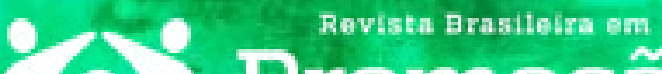

\section{ANÁLISE DO COMPORTAMENTO SEXUAL DE ADOLESCENTES}

\author{
Analysis of the sexual behavior of adolescents \\ Análisis de la conducta sexual de adolescentes
}

\author{
Laís Sandres Lins \\ Universidade Federal de Pernambuco - UFPE - Vitória de Santo Antão (PE) - Brasil.
}

\section{Luan Airton Marques da Silva}

Universidade Federal de Pernambuco - UFPE - Vitória de Santo Antão (PE) - Brasil.

\section{Robson Gomes dos Santos}

Universidade Federal de Pernambuco - UFPE - Vitória de Santo Antão (PE) - Brasil.

\section{Tayza Beatriz Duarte de Morais}

Universidade Federal de Pernambuco - UFPE - Vitória de Santo Antão (PE) - Brasil.

\section{Thaís de Andrade Beltrão}

Universidade Federal de Pernambuco - UFPE - Vitória de Santo Antão (PE) - Brasil.

\section{José Flávio de Lima Castro}

Universidade Federal de Pernambuco - UFPE - Vitória de Santo Antão (PE) - Brasil.

\section{RESUMO}

Objetivo: Analisar o comportamento sexual dos adolescentes, segundo o sexo, da cidade de Vitória de Santo Antão, Pernambuco. Métodos: Trata-se de um estudo descritivo, transversal e de abordagem quantitativa realizado com 282 adolescentes, de 12 a 19 anos, de ambos os sexos, em escolas públicas municipais de Vitória de Santo Antão, Pernambuco, Brasil. Os dados foram coletados no período de novembro de 2015 a fevereiro de 2016, através da aplicação de um questionário estruturado, na sala de aula e de forma individual, abordando questões sobre características sociodemográficas e comportamento sexual. A análise dos dados ocorreu com software SPSS utilizando a estatística descritiva e inferencial. Resultados: Os 282 adolescentes distribuíram-se em 170 (60,3\%) do sexo feminino e 112 (30,7 \%) do sexo masculino, com média de idade de $15,20(\mathrm{DP}= \pm 2,36)$ anos para os homens e $14,05(\mathrm{DP}= \pm 1,91)$ anos para as mulheres. Verificou-se que a iniciação sexual ocorreu com $41(57,7 \%)$ homens, com média de idade 14,44 (DP = $\pm 1,48)$ e $30(42,3 \%)$ mulheres, com média de $13,93(\mathrm{DP}= \pm 1,17)$ $(\mathrm{p}<0,001)$. Quanto ao preservativo masculino, identificou-se que $7(26,9 \%)$ das adolescentes e $19(73,1 \%)$ dos adolescentes utilizaram na primeira relação $(\mathrm{p}=0,018)$, e que $8(30,8 \%)$ das adolescentes e $18(69,2 \%)$ dos adolescentes na última relação ( $\mathrm{p}=0,045)$. Conclusão: Quanto ao comportamento sexual dos adolescentes, verificou-se que a iniciação sexual ocorreu de maneira precoce em ambos os sexos, sendo mais precocemente entre adolescentes do sexo masculino, e o método anticoncepcional mais utilizado durante o ato sexual foi o preservativo masculino. Sugere-se o desenvolvimento de estratégias na área da saúde e educação, favorecendo a troca de experiências entre os adolescentes, seus pais e a sociedade.

Descritores: Adolescente; Sexualidade; Comportamento Sexual.

\section{ABSTRACT}

Objective: To analyze the sexual behavior of adolescents, according to sex, in the city of Vitória de Santo Antão, PE. Methods: This is a descriptive, cross-sectional, quantitative study conducted with 282 adolescents aged 12 to 19 years, of both sexes, in municipal public schools in Vitória de Santo Antão, Pernambuco, Brazil. Data was collected from November 2015 to February 2016, through the application of a structured questionnaire, in the classroom on an individual basis, comprising questions on sociodemographic characteristics and sexual behavior. Data was analyzed by the software SPSS with use of descriptive and inferential statistics. Results: The 282 adolescents were distributed into 170 (60.3\%) females and 112 (30.7\%) males, with mean age of $15.20(S D= \pm 2.36)$ years for men and 14.05 (SD $= \pm 1.91)$ years for women. It was verified that sexual initiation occurred for $41(57.7 \%)$ men, at the mean age of $14.44(S D=1.48)$, and 30 (42.3\%) women, at the mean age of $13.93(S D= \pm 1.17)(p<0.001)$. As for the male condom, it was used by $7(26.9 \%)$ female adolescents and 19 (73.1\%) male adolescents in their first intercourse ( $p=0.018)$, and by $8(30.8 \%)$ female adolescents and $18(69.2 \%)$ male adolescents in their last intercourse ( $p=0.045)$. Conclusion: Regarding the sexual behavior of adolescents, it was verified that the sexual initiation occurred early in both genders, but earlier in male adolescents, and the contraceptive method most commonly used during the sexual act was the male 
condom. It is suggested that strategies be developed in the area of health and education, favoring the exchange of experiences among the adolescents, their parents and the society.

Descriptors: Adolescent; Sexuality; Sexual Behavior.

\section{RESUMEN}

Objetivo: Analizar la conducta sexual de los adolescentes según el sexo en la ciudad de Vitória de Santo Antão, Pernambuco. Métodos: Se trata de un estudio descriptivo, transversal y de abordaje cuantitativo realizado con 282 adolescentes entre 12 y 19 años de ambos sexos de escuelas públicas municipales de Vitória de Santo Antão, Pernambuco, Brasil. Se recogieron los datos en el período de noviembre de 2015 y febrero de 2016 a través de la aplicación de un cuestionario estructurado de manera individual con preguntas sobre las caracteristicas socio demográficas y la conducta sexual. Los datos fueron analizados con el software SPSS utilizando la estadistica descriptiva e inferencial. Resultados: De los 282 adolescentes, 170 (60,3\%) eran del sexo femenino y 112 (30,7\%) del sexo masculino con edad media de 15,20 (DS = $\pm 2,36)$ años para los hombres y 14,05 (DS = 1,91) años para las mujeres. Se verificó que la iniciación sexual se dio en $41(57,7 \%)$ hombres con edad media de 14,44 (DS = 1,48) años y $30(42,3 \%)$ años para las mujeres con edad media de $13,93(D S= \pm 1,17)(p<0,001)$. Respecto el preservativo masculino se identificó que $7(26,9 \%)$ de las adolescentes y $19(73,1 \%)$ de los adolescentes lo utilizaron en su primera relación sexual $(p=0,018)$ y $8(30,8 \%)$ de las adolescentes y 18 (69,2\%) de los adolescentes en la última relación ( $p=0,045)$. Conclusión: Respecto la conducta sexual de los adolescentes se verificó que la iniciación sexual se dio de manera precoz en ambos sexos y más precoz en el sexo masculino y el método anticonceptivo más utilizado durante la relación sexual ha sido el preservativo masculino. Se sugiere el desarrollo de estrategias en el área de la salud y la educación que favorezca el cambio de experiencias entre los adolescentes, sus padres y la sociedad.

Descriptores: Adolescente; Sexualidad; Conducta Sexual.

\section{INTRODUÇ̃̃̃O}

Segundo a Organização Mundial da Saúde (OMS), a adolescência é compreendida pela faixa etária variante de 10 a 19 anos $^{(1)}$. No Brasil, segundo o Estatuto da Criança e do Adolescente (ECA), a adolescência é considerada de 12 a 18 anos ${ }^{(2)}$. Nessa fase, ocorrem as principais modificações físicas, psicossociais e culturais, que vão desde o início da puberdade e maturação dos órgãos sexuais até o início do amadurecimento da identidade pessoal e sexual do indivíduo ${ }^{(3)}$.

Nesse período de transformações, ocorre frequentemente a experimentação da sexualidade, que, em um conceito mais amplo, é a energia que está contida no ser humano independente do sexo e da idade, a qual envolve práticas e desejos interligados a diferentes formas de sentir prazer e se satisfazer ${ }^{(4)}$.

No Brasil, a Pesquisa Nacional de Saúde do Escolar (PeNSE) de 2012 verificou que 29\% dos adolescentes de 13 a 15 anos já apresentaram a iniciação sexual, e que ainda, segundo o Ministério da Saúde, a média de idade da iniciação sexual, no Brasil, está ocorrendo aos $14,9 \operatorname{anos}^{(5)}$, sendo este momento marcado por diversas descobertas que irão ser fundamentais na definição do comportamento sexual do adolescente.

O comportamento sexual é um processo que ocorre em etapas e engloba diversos elementos, como orientação sexual, definindo se o adolescente é homossexual, heterossexual ou bissexual; a cultura e até mesmo circunstâncias de vida, e não se detém a ser uma característica fixa da sexualidade, podendo ser transitória e mutável no decorrer da vida ${ }^{(6)}$.

Além disso, o comportamento sexual está relacionado ao nível de conhecimento que o adolescente possui, pois irá refletir nas suas atitudes sexuais. Estudos demonstram que adolescentes que vivem em países em desenvolvimento geralmente possuem baixo conhecimento sexual, fato que repercutirá diretamente nas suas atitudes e nos seus padrões comportamentais e sexuais ${ }^{(7)}$.

O processo transitório da sexualidade, associado à iniciação precoce do ato sexual, vem mostrando que os adolescentes estão desenvolvendo comportamentos de risco, como o ato sexual desprotegido, associado ou não com o consumo de álcool, tabaco e outras drogas, levando às vulnerabilidades, as quais compreendem um conjunto de fatores, individuais ou coletivos, que podem expor esses adolescentes de maneira variável às infecções sexualmente transmissíveis/HIV e está diretamente ligada ao uso ou desuso de meios de proteção ${ }^{(8)}$.

A abordagem da temática vulnerabilidade deve acontecer na escola, pois é o local no qual o adolescente passa grande parte do seu tempo, sendo os professores importantes disseminadores do comportamento sexual seguro através da abordagem da educação sexual de modo transversal em todas as disciplinas ministradas. No entanto, verifica-se que os educadores estão despreparados para abordar essa temática e, quando o fazem, abordam apenas o enfoque biológico, desprezando a parte psicossocial e cultural ${ }^{9)}$.

Não se pode esquecer também do papel fundamental da família na construção da sexualidade e do comportamento sexual do(a) adolescente, pois eles devem se relacionar com os adolescentes de forma mais íntima, buscando a passagem de valores e práticas a partir do diálogo. Assim, a família pode ajudar a consolidar a formação do indivíduo, minimizando as atitudes de risco $^{(10)}$. 
As atitudes de risco(10), como, a falta do uso do preservativo e/ou dos anticoncepcionais, poderão acarretar graves consequências, seja a aquisição das infecções sexualmente transmissíveis/HIV, seja a gravidez indesejada, as quais influenciarão na vida dos adolescentes. A relação entre a atitude sexual de risco e a vulnerabilidade vem crescendo entre os adolescentes, tornando-se um grave problema de saúde pública.

Diante do exposto, pretende-se analisar o comportamento sexual dos adolescentes, segundo o sexo, da cidade de Vitória de Santo Antão, Pernambuco, visando que os resultados sirvam de subsídios para a criação e a implantação de políticas públicas que diminuam ou excluam as vulnerabilidades a que esse público possa estar exposto, minimizando as consequências negativas na vida dos adolescentes.

\section{MÉTODOS}

Trata-se de um estudo descritivo, transversal e de abordagem quantitativa, realizado com 282 adolescentes com idade de 12 a 19 anos, de ambos os sexos, matriculados nas escolas da rede pública municipal, do ensino fundamental e médio, da cidade de Vitória de Santo Antão - Pernambuco, Brasil, no período de novembro de 2015 a fevereiro de 2016.

A distribuição das escolas foi feita pela divisão de seu porte e período de matrícula dos estudantes (manhã, tarde e integral), visando garantir a proporcionalidade amostral. A fim de subsidiar o planejamento amostral, as escolas foram classificadas em três categorias: pequeno porte (menos de 200 alunos); médio porte (200 a 499 alunos); e grande porte (500 alunos ou mais). Todas as escolas da rede pública municipal de Vitória de Santo Antão foram consideradas elegíveis para inclusão no estudo.

Incluíram-se no estudo estudantes que atingiram os seguintes critérios: ser estudante do ensino fundamental ou médio, estar regularmente matriculado (a) na rede pública municipal de educação de Vitória de Santo Antão, Pernambuco, e, ter idade entre 12 a 19 anos.

Foram excluídos os estudantes que apresentaram alguma patologia neurológica ou alteração no estado físico, comportamental e/ou psicológico que impossibilitou o preenchimento dos instrumentos de coleta e aqueles que preencheram os questionários de forma errada.

Para seleção da amostra, recorreu-se ao procedimento de amostragem aleatória simples em dois estágios, em que a "escola" e a "turma" representaram as unidades amostrais, respectivamente, no primeiro e no segundo momento. Para a definição do número de escolas, utilizou a relação fornecida pela Secretaria Municipal de Educação de Vitória de Santo Antão e, para a turma, levou-se em consideração a densidade da turma com número médio de 25 alunos.

Após essas etapas, chegou-se ao número total de 9 escolas, sendo 4 de pequeno porte, 3 de médio e 2 de grande porte, correspondendo a 109 turmas. A randomização realizada no programa WinPepi representou 52,9\% das escolas municipais da cidade, totalizando uma amostra de 235 adolescentes.

Foram acrescidos $20 \%$ ao tamanho da amostra, visando atenuar as limitações por eventuais perdas na aplicação e/ou preenchimento inadequado dos questionários. Para o cálculo do tamanho amostral, foram adotados os seguintes parâmetros: população de 4.968 estudantes, intervalo de confiança de $95 \%$; erro máximo tolerável de 5 pontos percentuais. Por se tratar de estudo abrangendo a análise de múltiplos comportamentos de risco e com diferentes frequências de ocorrência, a prevalência estimada foi de $50 \%$, totalizando o quantitativo de 282 adolescentes.

Após aceitarem participar do estudo, os alunos levaram para casa o Termo de Consentimento Livre e Esclarecido para a sua assinatura e de seu responsável, e só após eram convidados a responder a um questionário estruturado auto-aplicável, o qual continha questões sobre características sociodemográficas e comportamento sexual. O instrumento foi baseado no questionário desenvolvido em uma tese da Universidade de São Paulo (USP) intitulada: Adolescência e vida sexual: análise do início da vida sexual de adolescentes residentes na zona leste do município de São Paulo ${ }^{(11)}$.

Os dados foram processados no programa Microsoft Excel 2010 e analisados utilizando o programa Statistical Package for the Social Sciences (SPSS), versão 13 (SPSS Inc., Chicago, IL, Estados Unidos da América, Release 16.0.2, 2008), sendo empregada a estatística descritiva com a distribuição de frequência e média com desvio-padrão.

$\mathrm{Na}$ análise inferencial, utilizou-se o mesmo programa, abordando os seguintes testes: o teste de Qui-quadrado, de Pearson, e o Exato, de Fisher, nas variáveis referentes aos métodos contraceptivos, prática sexual e ausência de vida sexual. No tocante às características sociodemográficas, utilizou-se, além desses dois, o Mann-Whitney. Nas variáveis acerca da orientação sexual, aplicou-se o Qui-quadrado, o Exato, o Mann-Whitney e o $t$-Student, com a finalidade de analisar as diferenças existentes entre os adolescentes segundo o sexo. Em todos os testes aplicados foi considerada significância estatística com o valor de $\mathrm{p} \leq 0,05$.

A presente pesquisa recebeu a aprovação do Comitê de Ética em Pesquisa da Universidade Federal de Pernambuco (CEP/ UFPE) com o parecer $n^{\circ} 1.268 .250$.

\section{RESULTADOS}

Os 282 adolescentes distribuíram-se em 170 (60,3\%) do sexo feminino e 112 (30,7\%) do sexo masculino, com média de idade de $15,20(\mathrm{DP}= \pm 2,36)$ anos para o sexo masculino e $14,05(\mathrm{DP}= \pm 1,91)$ anos para o sexo feminino, os quais apresentaram 
diferença estatisticamente significativa $(\mathrm{p}<0,001)$. Os dados referentes à caracterização das variáveis sociodemográficas e econômicas segundo o sexo podem ser vistos na Tabela I.

Tabela I - Distribuição das características sociodemográficas dos adolescentes. Vitória de Santo Antão, Pernambuco, 2016.

\begin{tabular}{|c|c|c|c|}
\hline \multirow[b]{2}{*}{ Variáveis } & \multicolumn{2}{|c|}{ Sexo } & \multirow[b]{2}{*}{ p-valor } \\
\hline & $\begin{array}{c}\text { Feminino } \\
\text { n }(\%)\end{array}$ & $\begin{array}{c}\text { Masculino } \\
\text { n (\%) }\end{array}$ & \\
\hline \multicolumn{4}{|l|}{ Cor } \\
\hline Parda & $102(64,2)$ & $57(35,8)$ & $0,156 *$ \\
\hline Branca & $27(50,9)$ & $26(49,1)$ & \\
\hline Amarela & $6(75,0)$ & $2(25,0)$ & \\
\hline Indígena & $2(28,6)$ & $5(71,4)$ & \\
\hline Preta & $22(64,7)$ & $12(35,3)$ & \\
\hline \multicolumn{4}{|l|}{ Religião } \\
\hline Católica & $87(61,7)$ & $54(38,3)$ & $0,113 *$ \\
\hline Evangélica & $55(66,3)$ & $28(33,7)$ & \\
\hline Espírita & $0(0,0)$ & $3(100,0)$ & \\
\hline Nenhuma & $22(53,7)$ & $19(46,3)$ & \\
\hline Outra & $3(42,9)$ & $4(57,1)$ & \\
\hline \multicolumn{4}{|l|}{ Pessoa religiosa } \\
\hline Sim & $80(66,7)$ & $40(33,3)$ & $0,164 * *$ \\
\hline Não & $15(57,7)$ & $11(42,3)$ & \\
\hline Mais ou menos & $75(55,1)$ & $61(44,9)$ & \\
\hline \multicolumn{4}{|l|}{ Período de estudo } \\
\hline Manhã & $73(61,3)$ & $46(38,7)$ & $0,244 * *$ \\
\hline Tarde & $94(61,0)$ & $60(39,0)$ & \\
\hline Integral & $3(33,3)$ & $6(66,7)$ & \\
\hline \multicolumn{4}{|c|}{ Trabalho assalariado } \\
\hline Sim & $10(37,0)$ & $17(63,0)$ & $0,009 * *$ \\
\hline Não & $160(62,7)$ & $95(37,3)$ & \\
\hline \multicolumn{4}{|l|}{ Tipo de moradia } \\
\hline Alugada & $48(64,9)$ & $26(35,1)$ & $0,402 * *$ \\
\hline Própria & $118(59,3)$ & $81(40,7)$ & \\
\hline \multicolumn{4}{|l|}{ Procedência } \\
\hline Zona urbana & $108(56,5)$ & $83(43,5)$ & $0,511 * *$ \\
\hline Zona rural & $38(61,3)$ & $24(38,7)$ & \\
\hline \multicolumn{4}{|l|}{ Renda mensal } \\
\hline Menos de 1 SM & $47(62,7)$ & $28(37,3)$ & $0,192 * *$ \\
\hline Entre 1 e 2 SM & $26(57,8)$ & $19(42,2)$ & \\
\hline \multirow[t]{2}{*}{ Mais de 2 SM } & $15(44,1)$ & $19(55,9)$ & \\
\hline & Média \pm DP & Média \pm DP & \\
\hline Idade (anos) & $14,05 \pm 1,91$ & $15,20 \pm 2,36$ & $<0,001 * * *$ \\
\hline
\end{tabular}

(*) Exato de Fisher; (**) Qui-Quadrado; (***) Mann-Whitney; SM = salários mínimos

Quanto à orientação sexual e iniciação sexual, observou-se que houve diferença nas variáveis iniciação sexual, decisão de ter coitarca, local da coitarca e idade do parceiro na $1^{a}$ vez, como pode visto ser na Tabela II. 
Tabela II - Distribuição das variáveis relacionadas à orientação sexual e primeira relação sexual. Vitória de Santo Antão, Pernambuco, 2016.

\begin{tabular}{|c|c|c|c|}
\hline \multirow[b]{2}{*}{ Variáveis } & \multicolumn{2}{|c|}{ Sexo } & \multirow[b]{2}{*}{ p-valor } \\
\hline & $\begin{array}{c}\text { Feminino } \\
\text { n (\%) }\end{array}$ & $\begin{array}{c}\text { Masculino } \\
\text { n (\%) }\end{array}$ & \\
\hline \multicolumn{4}{|l|}{ Iniciação sexual } \\
\hline $\operatorname{Sim}$ & $30(42,3)$ & $41(57,7)$ & $<0,001 * *$ \\
\hline Não & $140(66,4)$ & $71(33,6)$ & \\
\hline \multicolumn{4}{|l|}{ Coitarca } \\
\hline Mesmo sexo & $3(37,5)$ & $5(63,5)$ & $0,691 *$ \\
\hline Sexo oposto & $27(42,9)$ & $36(57,1)$ & \\
\hline \multicolumn{4}{|l|}{ Decisão de ter coitarca } \\
\hline Atração & $4(22,2)$ & $14(77,8)$ & 0,046 \\
\hline Outro & $0(0,0)$ & $4(100,0)$ & \\
\hline Paixão/amor & $11(64,7)$ & $6(35,3)$ & \\
\hline Pressão do(a) companheiro(a) & $1(100,0)$ & $0(0,0)$ & \\
\hline Queria perder a virgindade & $5(50,0)$ & $5(50,0)$ & \\
\hline Pressão dos amigos & $0(0,0)$ & $1(100,0)$ & \\
\hline Curiosidade & $9(45,0)$ & $11(55,0)$ & \\
\hline \multicolumn{4}{|l|}{ Sentimento durante coitarca } \\
\hline Dor & $2(100,0)$ & $0(0,0)$ & $0,069 *$ \\
\hline Outro & $0(0,0)$ & $2(100,0)$ & \\
\hline Nervosismo & $9(50,0)$ & $9(50,0)$ & \\
\hline Excitação & $5(29,4)$ & $12(70,6)$ & \\
\hline Medo & $2(66,7)$ & $1(33,3)$ & \\
\hline Prazer & $6(33,3)$ & $12(66,7)$ & \\
\hline Dor / Nervosismo & $2(100,0)$ & $0(0,0)$ & \\
\hline Excitação / Prazer & $0(0,0)$ & $3(100,0)$ & \\
\hline Dor / Nervosismo / Medo & $2(100,0)$ & $0(0,0)$ & \\
\hline Dor / Medo / Prazer & $2(100,0)$ & $0(0,0)$ & \\
\hline Nervosismo / Excitacão / Prazer & $0(0,0)$ & $2(100,0)$ & \\
\hline \multicolumn{4}{|l|}{ Local da coitarca } \\
\hline Na sua casa & $4(16,7)$ & $20(83,3)$ & $0,001 *$ \\
\hline Outro & $2(66,7)$ & $1(33,3)$ & \\
\hline Na casa dele (a) & $19(70,3)$ & $8(29,7)$ & \\
\hline Na casa de amigos & $3(37,5)$ & $5(62,5)$ & \\
\hline Motel $/$ hotel & $2(66,7)$ & $1(33,3)$ & \\
\hline Carro & $0(0,0)$ & $3(100,0)$ & \\
\hline Lugar de prostituição & $0(0,0)$ & $1(100,0)$ & \\
\hline \multirow{2}{*}{ Espaço público (rua, parque) } & $0(0,0)$ & $2(100,0)$ & \\
\hline & Média \pm DP & Média \pm DP & \\
\hline Idade na coitarca & $13,93 \pm 1,17$ & $14,44 \pm 1,48$ & $0,119 * * *$ \\
\hline Idade do parceiro na $1^{\mathrm{a}} \mathrm{vez}$ & $17,46 \pm 3,00$ & $15,20 \pm 2,20$ & $0,003 \S$ \\
\hline
\end{tabular}

(*) Exato de Fisher; (**) Qui-Quadrado; (***) Mann-Whitney; (§) t Student. 
A Tabela III retrata o uso dos métodos contraceptivos e a prática sexual dos adolescentes. Com base nos resultados apresentados, verificou-se que houve diferença na comparação entre os sexos na maioria das variáveis avaliadas.

Tabela III - Distribuição das variáveis relacionadas ao emprego de métodos contraceptivos e prática sexual dos adolescentes na primeira e na última relação sexual. Vitória de Santo Antão, Pernambuco, 2016.

\begin{tabular}{|c|c|c|c|}
\hline \multirow[b]{2}{*}{ Variáveis } & \multicolumn{2}{|c|}{ Sexo } & \multirow[b]{2}{*}{ p-valor } \\
\hline & $\begin{array}{c}\text { Feminino } \\
\text { n }(\%)\end{array}$ & $\begin{array}{c}\text { Masculino } \\
\text { n (\%) }\end{array}$ & \\
\hline \multicolumn{4}{|c|}{$\begin{array}{l}\text { Métodos contraceptivos e de barreira na primeira } \\
\text { relação sexual }\end{array}$} \\
\hline Sim & $21(47,7)$ & $23(52,3)$ & $0,398 * *$ \\
\hline Não & $9(33,3)$ & $18(66,6)$ & \\
\hline \multicolumn{4}{|l|}{ Se sim, qual método utilizado? } \\
\hline Tabelinha & $2(66,7)$ & $1(33,3)$ & $0,018 *$ \\
\hline Pílula & $4(80,0)$ & $1(20,0)$ & \\
\hline Preservativo masculino & $9(32,1)$ & $19(67,9)$ & \\
\hline Camisinha feminina & $1(50,0)$ & $1(50,0)$ & \\
\hline Injeção & $5(83,3)$ & $1(16,7)$ & \\
\hline \multicolumn{4}{|c|}{ Caso não tenha usado camisinha, por que não usou } \\
\hline Não conhecia & $0(0,0)$ & $6(100,0)$ & $0,006 *$ \\
\hline Não esperava ter relação naquele dia & $3(37,5)$ & $5(62,5)$ & \\
\hline Queria engravidar & $1(100,0)$ & $0(0,0)$ & \\
\hline Conhecia bem o(a) companheiro(a) & $0(0,0)$ & $3(100,0)$ & \\
\hline Estava muito apaixonada (o) & $1(100,0)$ & $0(0,0)$ & \\
\hline Companheiro (a) não aceitou & $1(100,0)$ & $0(0,0)$ & \\
\hline Nem pensou sobre isto & $1(100,0)$ & $0(0,0)$ & \\
\hline Não gosta & $1(33,3)$ & $2(66,7)$ & \\
\hline Não tinha camisinha no momento & $1(33,3)$ & $2(66,7)$ & \\
\hline \multicolumn{4}{|c|}{$\begin{array}{l}\text { Utilizou algum método contraceptivo na última } \\
\text { relação }\end{array}$} \\
\hline Sim & $22(45,8)$ & $26(54,2)$ & $0,190 * *$ \\
\hline Não & $3(25,0)$ & $9(75,0)$ & \\
\hline \multicolumn{4}{|l|}{ Se sim, qual método? } \\
\hline Tabelinha & $2(100,0)$ & $0(0,0)$ & $0,045 *$ \\
\hline Pílula & $7(63,6)$ & $4(36,4)$ & \\
\hline Preservativo masculino & $8(29,6)$ & $19(70,4)$ & \\
\hline Camisinha feminina & $0(0,0)$ & $1(100,0)$ & \\
\hline Injeção & $5(71,4)$ & $2(28,6)$ & \\
\hline \multicolumn{4}{|c|}{ Quanto a sua orientação sexual, você se considera } \\
\hline Homossexual & $0(0,0)$ & $3(100,0)$ & $0,180 *$ \\
\hline Heterossexual & $18(41,9)$ & $25(58,1)$ & \\
\hline Bissexual & $3(75,0)$ & $1(25,0)$ & \\
\hline \multicolumn{4}{|c|}{ Quantos parceiros você já se envolveu até hoje } \\
\hline $\mathrm{Um}$ & $12(75,0)$ & $4(25,0)$ & $0,035 *$ \\
\hline Dois & $7(58,3)$ & $5(41,7)$ & \\
\hline Três & $4(36,4)$ & $7(63,6)$ & \\
\hline Quatro & $2(33,3)$ & $4(66,7)$ & \\
\hline Cinco ou mais & $1(12,5)$ & $7(87,5)$ & \\
\hline
\end{tabular}

(*) Exato de Fisher; (**) Qui-Quadrado

No tocante à ausência da iniciação sexual, verificou-se diferença estatística significativa em relação ao sexo, observando-se motivações diferentes a depender do sexo, como verificado na Tabela IV. 
Tabela IV - Distribuição das variáveis relacionadas à ausência de vida sexual. Vitória de Santo Antão, Pernambuco, 2016.

\begin{tabular}{|c|c|c|c|}
\hline \multirow[b]{2}{*}{ Variáveis } & \multicolumn{2}{|c|}{ Sexo } & \multirow[b]{2}{*}{ p-valor } \\
\hline & $\begin{array}{c}\text { Feminino } \\
\text { n }(\%)\end{array}$ & $\begin{array}{c}\text { Masculino } \\
\text { n }(\%) \\
\end{array}$ & \\
\hline \multicolumn{4}{|l|}{ Ausência da iniciação sexual } \\
\hline Quer casar virgem & $39(69,6)$ & $17(30,4)$ & $<0,001 *$ \\
\hline Outro & $2(50,0)$ & $2(50,0)$ & \\
\hline Ainda não encontrou a pessoa certa & $20(48,8)$ & $21(51,2)$ & \\
\hline Não teve oportunidade & $4(30,8)$ & $9(69,2)$ & \\
\hline Tem medo de engravidar & $12(80,0)$ & $3(20,0)$ & \\
\hline Ainda é muito novo (a) & $62(79,5)$ & $16(20,5)$ & \\
\hline Tem medo de DST/AIDS & $1(33,3)$ & $2(66,7)$ & \\
\hline \multicolumn{4}{|l|}{ A maioria dos seus amigos é virgem } \\
\hline $\operatorname{Sim}$ & $67(74,4)$ & $23(25,6)$ & $0,715 * *$ \\
\hline Não & $30(71,4)$ & $12(28,6)$ & \\
\hline
\end{tabular}

(*) Exato de Fisher; (**) Qui-Quadrado

\section{DISCUSSÃO}

Observou-se que a média de idade dos adolescentes investigados no presente estudo foi similar ao encontrado em outra pesquisa sobre a mesma temática, onde se encontrou média de idade de $14,44(\mathrm{DP}= \pm 1,48)$ anos para o sexo masculino e 13,93 $(\mathrm{DP}= \pm 1,17)$ para o sexo feminino ${ }^{(12)}$.

Verificou-se na atual pesquisa diferença também quanto ao trabalho assalariado, contatando-se que os adolescentes do sexo masculino trabalhavam e as adolescentes não possuíam renda. Esse achado se encontra em consonância com o estudo por amostra de domicílio com 91.377 crianças e adolescentes de todo território brasileiro, que revelou, quando comparados trabalhadores e não trabalhadores, a maior proporção de trabalhadores do sexo masculino, com faixa etária de 14 a 17 anos, pretos e pardos ${ }^{(13)}$. Tal fato pode estar associado à relação de gênero, pois as mulheres são criadas com uma educação voltada para os trabalhos domésticos, enquanto a do homem está voltada para o trabalho fora de casa e remunerado, contribuindo para a renda familiar ${ }^{(14)}$, e, de modo geral, o perfil dos adolescentes estudados é de baixa renda.

A baixa renda é um fato marcante em todo o país, segundo dados do Instituto Brasileiro de Geografia e Estatística (IBGE) em seu relatório sobre a situação da adolescência brasileira, sendo elevado o percentual de adolescentes de 12 a 17 anos vivendo em famílias extremamente pobres (até 1/4 salário mínimo) em todo o Brasil. O Nordeste é o estado que concentra as piores prevalências, sendo o estado de Pernambuco o quarto pior do Brasil, com $32 \%$, perdendo apenas para os estados de Alagoas $(38,4 \%)$, Maranhão $(33,1 \%)$ e Piauí $(32,8 \%)^{(15)}$. Dessa forma, pode-se verificar que os adolescentes analisados no presente estudo estão inseridos em um grupo com inserção social desfavorecida, não permitindo, portanto, generalizar os dados encontrados para adolescentes de outros níveis sociais.

Quanto à iniciação sexual dos adolescentes, observou-se na atual pesquisa que houve diferença entre os gêneros, com maior prevalência do sexo masculino em relação ao feminino. No entanto, são frequentes as variações em relação às prevalências de iniciação sexual, como se observou em outros estudos. O primeiro, desenvolvido em três escolas estaduais de ensino fundamental e médio do município de Embu, São Paulo, constatou prevalência de 39\% no sexo masculino e $17 \%$ no feminino ${ }^{(16)}$.

O segundo, realizado com 136 indivíduos de 15 a 18 anos de idade, estudantes de uma escola pública da cidade de Bauru, São Paulo, revelou que há maior porcentagem de homens $(52,1 \%)$ do que de mulheres $(47,9 \%)$ que tiveram relação sexual, o que difere dos dados do IBGE, que apontam $43,7 \%$ entre os homens e $18,7 \%$ entre as mulheres ${ }^{(17)}$, assim como, de estudos internacionais que também apontam essa divergência, demonstrando percentuais de $41,7 \%$ para os adolescentes e $31,8 \%$ para as adolescentes em Portugal ${ }^{(18)}$; e de 47,1\% para os adolescentes e 35,2\% para as adolescentes em Hong Kong ${ }^{(19)}$.

No Brasil, essa maior prevalência da iniciação sexual dos adolescentes masculinos, justifica-se, possivelmente, pela forma de criação distinta entre os sexos, pois os adolescentes precisam comprovar a sua masculinidade, para a família e a sociedade, levando ao precoce interesse masculino ${ }^{(20)}$. Em contrapartida, para o sexo feminino, a sexualidade é atribuída à reprodução, devendo ser suprimida antes do casamento, postergando sua iniciação sexual ${ }^{(21)}$.

Diante do fato social da iniciação sexual, percebeu-se, no atual estudo, que a decisão da coitarca apresentou diferenças quanto ao seu significado, pois, para o sexo masculino, a decisão foi por atração e curiosidade, diferente do sexo feminino, que relatou a paixão/amor. Pode-se destacar, com isso, que para se envolverem sexualmente, as adolescentes precisam ter um vínculo, como o amor e paixão; já o romantismo não é valorizado pelos adolescentes, pois, para a iniciação sexual, apenas a atração é suficiente( ${ }^{(22)}$. 
Quanto ao local da coitarca, a casa do adolescente foi o mais frequente no presente estudo. Esse achado pode estar associado ao fato das adolescentes serem reprimidas socialmente e pela família, fazendo com que tenham um comportamento baseado no temor da "descoberta" de estar fazendo algo que a levam a acreditar ser incorreto ${ }^{(20)}$.

A idade dos parceiros na primeira relação sexual apresentou diferença entre os adolescentes do estudo em questão. Apesar de ambos os sexos se relacionarem com pessoas mais velhas, as adolescentes apresentaram maior diferença de idade quando comparadas aos adolescentes. Esses resultados mostram a realidade brasileira e reforçam o padrão das adolescentes se envolverem com parceiros mais velhos e experientes ${ }^{(12)}$.

Quanto ao emprego de métodos contraceptivos e de barreira na primeira relação, também se observou diferença entre os sexos no presente estudo. O sexo masculino apresentou maiores índices em relação ao feminino, e o método mais prevalente adotado, por ambos, foi o preservativo masculino. Quando comparada a última relação sexual, verificou-se um aumento na prevalência, em ambos, de uso do preservativo. Essa diferença quanto ao uso do preservativo entre os sexos pode estar relacionada ao sentimento de vergonha ao descobrir preservativos dentro da bolsa, como também na confiança no(a) parceiro(a), com isso, todos esses fatores deixam as mulheres mais vulneráveis às infecções sexualmente transmissíveis, HIV e gravidez na adolescência ${ }^{(23)}$.

No tocante ao número de parceiros, também houve diferença entre os gêneros, constatando-se que os adolescentes tiveram três ou mais parceiras na presente pesquisa. A maioria das adolescentes só teve relação com um parceiro. Provavelmente, tal fato possa ser explicado em razão dos adolescentes supervalorizarem a sua sexualidade, buscando mostrar para a sociedade sua masculinidade, enquanto as adolescentes não se sentem à vontade para relatarem o número correto de parceiros, pois serão recriminadas $^{(24)}$.

Dentre os adolescentes que responderam não ter vida sexual, constatou-se, na presente pesquisa, diferença segundo o gênero, observando que, para as adolescentes, questões como "ser muito nova" e "querer casar virgem" contribuíram para a não ocorrência da relação sexual, enquanto para os adolescentes, o fato que mais colaborou é não ter encontrado a pessoa certa. Assim, mais uma vez, fica evidente a influência de valores sociais, já que a iniciação das atividades sexuais nos adolescentes do sexo masculino é mais aceita ${ }^{(21)}$.

Algumas limitações precisam ser relatadas em relação ao presente estudo, como a amostra específica de uma região do Brasil, não se podendo deduzir que as conclusões encontradas sejam aplicáveis em outras regiões brasileiras ou mesmo mundialmente. Além disso, todas as respostas foram baseadas no autorrelato dos adolescentes por meio de questionários autoaplicáveis, o que pode ocasionar um viés de memória, uma fragilidade inerente aos estudos transversais retrospectivos. Também se devem considerar as dificuldades relacionadas ao público de adolescentes, visto que as adolescentes tendem a subestimar as experiências sexuais, enquanto os adolescentes tendem a superestimar ${ }^{(24)}$.

O presente estudo também pôde verificar a influência sociocultural nas condutas e práticas dos adolescentes, averiguandose um padrão de valores distintos entre os sexos, que acabam por afetar o comportamento sexual desse grupo. Percebe-se, portanto, a necessidade de desenvolvimento de estratégias pelas equipes de saúde e pelos professores em relação à educação em saúde nas escolas, favorecendo a troca de experiências entre os adolescentes, seus pais e a sociedade quanto à igualdade entre os sexos.

Por fim, ficam como sugestões a realização de estudos transversais e longitudinais futuros, os quais comparem as diferentes realidades brasileiras, as diversidades entre os estudantes do ensino privado e público, e avaliem outras atitudes de risco que possam afetar os aspectos sexuais e reprodutivos, como uso do álcool e drogas.

\section{CONCLUSÃO}

Quanto ao comportamento sexual dos adolescentes, verificou-se que a iniciação sexual ocorreu de maneira precoce em ambos os sexos, sendo mais precocemente entre adolescentes do sexo masculino, e o método anticoncepcional mais utilizado durante o ato sexual foi o preservativo masculino. Sugere-se o desenvolvimento de estratégias na área da saúde e educação, favorecendo a troca de experiências entre os adolescentes, seus pais e a sociedade.

\section{REFERÊNCIAS}

1. Bechara AMD, Gontijo DT, Medeiros M, Facundes VLD. Na brincadeira a gente foi aprendendo: promoção de saúde sexual e reprodutiva com homens adolescentes. Rev Eletrônica Enferm [Internet]. 2013 [acesso em 2016 Fev 04];15(1):2533. Disponível em: https://revistas.ufg.emnuvens.com.br/fen/article/view/19046/14042

2. Trabbold VLM, Caleiro RCL, Cunha CF, Guerra AMC. Concepções sobre adolescentes em situação de violência sexual. Psicol Soc [Internet]. 2016 [acesso em 2016 Fev 04];28(1):499-506. Disponível em: http://www.scielo.br/scielo. php?script=sci_arttext\&pid=S0102-71822016000100074\&lang=pt 
3. Albuquerque JG, Pinheiro PNC, Lopes, MVO, Machado MFAS. Conhecimento deficiente acerca do HIV/AIDS em estudantes adolescentes: identificação de diagnóstico de enfermagem NANDA. Rev Eletrônica Enferm [Internet]. 2012 [acesso em 2016 Fev 04];14(1):104-11. Disponível em: http://www.revistas.ufg.br/index.php/fen/article/view/12256

4. Macedo SRH, Miranda FAN, Pessoa JMJ, Nóbrega VKM. Adolescência e sexualidade: scripts sexuais a partir dasrepresentações sexuais. Rev Bras Enferm [Internet]. 2013 [acesso em 2016 Fev 04];66(1):103-9. Disponível em: http:// www.scielo.br/scielo.php?script=sci arttext\&pid=S0034-71672013000100016

5. Gonçalves H, Machado IEC, Soares ALG, Camargo-Figuera FA, Seerig LM, Mesenburg MA. Início da vida sexual entre adolescentes (10 a 14 anos) e comportamentos em saúde. Rev Bras Epidemiol [Internet]. 2015 [acesso em $2016 \mathrm{Fev}$ 04];18(1):499-506. Disponível em: http://www.scielo.br/scielo.php?script=sci_arttext\&pid=S1415790X2015000100025 \&lng=pt

6. Assis SG, Gomes R, Pires TO. Adolescência, comportamento sexual e fatores de risco à saúde. Rev Saúde Pública [Internet]. 2014 [acesso em 2016 Fev 04];48(1). Disponível em: http://www.scielo.br/scielo.php?pid=S003489102014000100043\&sc ript $=$ sci_abstract\&tlng $=$ pt

7. Zhou H, Wang XY, Ye F, Gu HH, Zeng XP, Wang Y. Contraceptive knowledge, atitudes and behavior about sexuality among college students in Beijing, China. Chin Med J (Engl) [Internet]. 2012 [acesso em 2016 Fev 04];125(6):1153-7. Disponível em: http://www.ncbi.nlm.nih.gov/pubmed/22613546

8. Moraes SP, Vitalle MSS. Direitos sexuais e reprodutivos na adolescência. Rev Assoc Med Bras [Internet]. 2012 [acesso em 2016 Fev 04];58(1):48-52. Disponível em: http://www.scielo.br/pdf/ramb/v58n1/v58n1a14.pdf

9. Oliveira-Campos M, Nunes ML, Madeira FC, Santos MG, Bregmann SR, Malta DC, et al. Sexual behavior among Brazilian adolescents, National Adolescent School-based Health Survey (PeNSE 2012). Rev Bras Epidemiol [Internet]. 2014 [acesso em 2016 Fev 04]; 17(Supl):116-30. Disponível em: http://www.scielosp.org/pdf/rbepid/v17s1/1415-790Xrbepid-17-s1-00116.pdf

10. Gonçalves RC, Faleiro JH, Malafaia G. Educação sexual no contexto familiar e escolar: impasses e desafios. Holos [Internet] 2013 [acesso em 2016 Fev 04];5(29):251-63. Disponível em: http://www2.ifrn.edu.br/ojs/index.php/HOLOS/ article/view/784

11. Borges BLV. Adolescência e vida sexual: analise do início da vida sexual de adolescentes residentes na zona leste do município de São Paulo [tese]. São Paulo: Universidade de São Paulo; 2004 [acesso em 2016 Fev 04]. Disponível em: http://www.teses.usp.br/teses/disponiveis/6/6136/tde-15042005-112703/pt-br.php

12. Hugo TDO, Maier VT, Jansen K, Rodrigues CEG, Cruzeiro ALS, Ores LC, et al. Fatores associados à idade da primeira relação sexual em jovens: estudo de base populacional. Cad Saúde Pública [Internet]. 2011 [acesso em 2016 Fev 04];27(11):2207-14. Disponível em: http://www.scielosp.org/pdf/csp/v27n11/14.pdf

13. Miquilin IOC, Marín-León L, Luz VG, La-Rotta EIG, Corrêa HR Filho. Perfil demográfico, socioeconômico e de saúde de crianças e adolescentes trabalhadores e não trabalhadores, Brasil: análise das desigualdades. Cad Saúde Pública [Internet]. 2015 [acesso em 2016 Fev 04];31(9). Disponível em: http://www.scielosp.org/scielo.php?script=sci_arttext\&pid=S01023 $11 \mathrm{X} 2015001001856 \&$ lang $=\mathrm{pt}$

14. Cecilio SG, Silveira RCP. Caracterização do trabalho de menores de uma escola estadual de Divinópolis-MG. Cienc Enferm [Internet]. 2014 [acesso em 2016 Fev 04];20(1):47-60. Disponível em: http://www.scielo.cl/scielo.php?script=sci_ arttext\&pid $=$ S071795532014000100005\&lang $=$ pt

15. United Nations International Children's Emergency Fund - UNICEF. O direito de ser adolescente: oportunidade para reduzir vulnerabilidades e superar desigualdades [Internet]. Brasília: UNICEF; 2011 [acesso em 2016 Fev 04]. Disponível em: http://www.unicef.org/brazil/pt/br_sabrep11.pdf

16. Brêtas JRS, Ohara CVS, Jardim DP, Aguiar W Junior, Oliveira JR. Aspectos da sexualidade na adolescência. Ciênc Saúde Coletiva [Internet]. 2011 [acesso em 2016 Fev 04];16(7):3221-8. Disponível em: http://www.scielo.br/scielo. php?script $=$ sci_arttext\&pid $=$ S1413-81232011000800021

17. Senem CJ, Correr R, Costa FM Junior, Caramachi S, Vasconcellos S. Vulnerabilidade ao HIV em estudantes de ensino médio de uma escola pública no interior de São Paulo. SALUSVITA [Internet]. 2014 [acesso em 2016 Fev 04];33(1):4555. Disponível em: http://www.usc.br/biblioteca/salusvita/salusvita_v33_n1_2014_art_03.pdf

18. Ferreira MMSRS, Torgal MCLFPR. Estilos de vida na adolescência: comportamento sexual dos adolescentes portugueses. Rev Esc Enferm USP [Internet]. 2011 [acesso em 2016 Fev 04]; 45(3):589-95. Disponível em: http://www.scielo.br/pdf/ reeusp/v45n3/v45n3a06.pdf 
19. Yip PSF, Zhang H, Lam TH, Lam KF, Lee AM, Chand J, et al. Sex knowledge, attitudes, and high-risk sexual behaviors among um married youth in Hong Kong. BMC Public Health [Internet]. 2013 [acesso em 2016 Fev 04];13:691. Disponível em: http://www.biomedcentral.com/1471-2458/13/691

20. Ressel LB, Junges CF, Sehenem GD, Sanfelice C. A influência da família na vivência da sexualidade de mulheres adolescentes. Esc Anna Nery Rev Enferm [Internet]. 2011 [acesso em 2016 Fev 04];15(2):245-50. Disponível em: http:// www.scielo.br/pdf/ean/v15n2/v15n2a05.pdf

21. Anjos RHD, Silva JAS, Val LF, Rincon LA, Nichiata LYI. Diferenças entre adolescentes do sexo feminino e masculino na vulnerabilidade individual ao HIV. Rev Esc Enferm USP [Internet]. 2012 [acesso em 2016 Fev 04];46(4):829-37. Disponível em: http://www.scielo.br/scielo.php?script=sci_arttext\&pid=S0080-62342012000400007

22. Santos TMB, Albuquerque LBB, Bandeira CF, Colares VSA. Fatores que contribuem para o início da atividade sexual em adolescentes: revisão integrativa. Rev Atenção Saúde [Internet]. 2015 [acesso em 2016 Fev 04];13(44):64-70. Disponível em: http://seer.uscs.edu.br/index.php/revista_ciencias_saude/article/view/2668/1740

23. Ruivo PVA, Gomes G, Xavier DM, Costa SS. Vivências de pais adolescentes com o uso de métodos contraceptivos. Rev Enferm UFPE Online [Internet]. 2014 [acesso em 2016 Fev 04];8(2):249-56. Disponível em: http:/www.revista.ufpe.br/ revistaenfermagem/index.php/revista/article/download/8974/14159

24. Coutinho RZ, Miranda-Ribeiro P. Religião, religiosidade e iniciação sexual na adolescência e juventude: lições de uma revisão bibliográfica sistemática de mais de meio século de pesquisas. Rev Bras Estud Popul [Internet]. 2014 [acesso em 2016 Fev 04];31(2):333-65. Disponível em: http://www.scielo.br/scielo.php?script=sci_arttext\&pid=S0102$30982014000200006 \&$ lang $=$ pt

\section{Endereço para correspondência:}

Laís Sandres Lins

Universidade Federal de Pernambuco - Centro Acadêmico de Vitória

Rua: Alto do Reservatório, S/N

Bairro: Bela Vista

CEP: 55608-680 - Vitória de Santo Antão (PE) - Brasil

E-mail: lais-sandres@hotmail.com 